\title{
Flux de calcium et contrôle de la glycogénolyse hépatique
}

Dans une cellule non stimulée, la concentration cytosolique de calcium est faible alors qu'elle est $\mathbf{1 0} \mathbf{0 0 0}$ fois plus élevée dans le milieu extracellulaire et que ce cation est stocké dans le réticulum endoplasmique et les mitochondries. Certaines hormones mobilisent très rapidement le calcium des réserves du réticulum endoplasmique, via la production d'inositol triphosphate ; la persistance d'une concentration élevée de calcium dans le cytosol exige un influx augmenté à partir du milieu extracellulaire. Le calcium se comporte dans la cellule comme un messager de l'action hormonale, modifiant l'activité de divers systèmes enzymatiques.

Jean-Pierre Mauger

Chargé de recherche à l'Inserm

\section{Michel Claret}

Directeur de recherche au Cnrs

\section{ADRESSE}

J.-P. Mauger, M. Claret : unité de recherches de physiologie et pharmacologie cellulaire, Inserm U.274, bâtiment 443, université Paris- e métabolisme des glucides dans le foie èt dans les muscles est contrôlé par des hormones circulantes et par des neuromédiateurs. Le signal, reconnu au niveau de la membrane plasmique par des récepteurs spécifiques, est transmis à l'intérieur de la cellule cible par des messagers intracellulaires. L'AMP cyclique découvert en 1958 par Sutherland [1] est le messager intracellulaire actuellement le mieux connu. L'AMP cyclique active une protéine kinase qui entraîne la phosphorylation de certaines protéines dans la cellule. Le transfert d'un groupe phosphate sur une protéine induit un changement de conformation et permet de modifier ses propriétés [2]. Ainsi, par exemple, la phosphorylase bkinase, qui est l'enzyme clé contrôlant la dégradation du glycogène est activée par phosphorylation lorsque la concentration d'AMP cyclique augmente. Cette voie utilisant l'AMP cyclique comme second messager est mise en jeu, dans le foie, par plusieurs hormones comme le glucagon ou l'adrénaline lorsque cette dernière interagit avec des récepteurs $\beta$-adrénergiques.

Cependant, l'AMP cyclique n'est pas le seul messager intracellulaire impliqué dans la régulation du métabolisme cellulaire. Un certain nombre d'hormones ou de neuromédiateurs produisent leurs effets sur les cellules cibles sans modifier le contenu cellulaire en AMP cyclique. Ainsi, les catécholamines, en stimulant les récepteurs $\alpha_{1}$-adrénergiques, ou des hormones vaso-actives comme l'angiotensine ou la vasopressine, augmentent la concentration de $\mathrm{Ca}^{2+}$ ionisé dans les hépatocytes. De plus, il a été montré que les ionophores à $\mathrm{Ca}^{2+}$, qui accélèrent les échanges de $\mathrm{Ca}^{2}+$ à travers les membranes cellulaires, reproduisent l'effet stimulateur des hormones sur la glycogénolyse. Inversement, si des hépatocytes ont été "déplétés " de leur calcium, les hormones indépendantes de l'AMP cyclique n'activent 
Figure 1. Mesure du $\mathrm{Ca}^{2+}$ cytosolique par lo quin 2. Le quin 2 acétoxyméthylester (quin 2/AM) diffuse librement à travers la membrane plasmique. Les estérases présentes dans le cytosol des cellules hydrolysent les liaisons esters (COX) et rendent le quin 2 libre (COO-) apte à fixer le $\mathrm{Ca}^{2+}$. Le complexe quin 2-Ca ${ }^{2+}$ entraîne une émission de fluorescence.

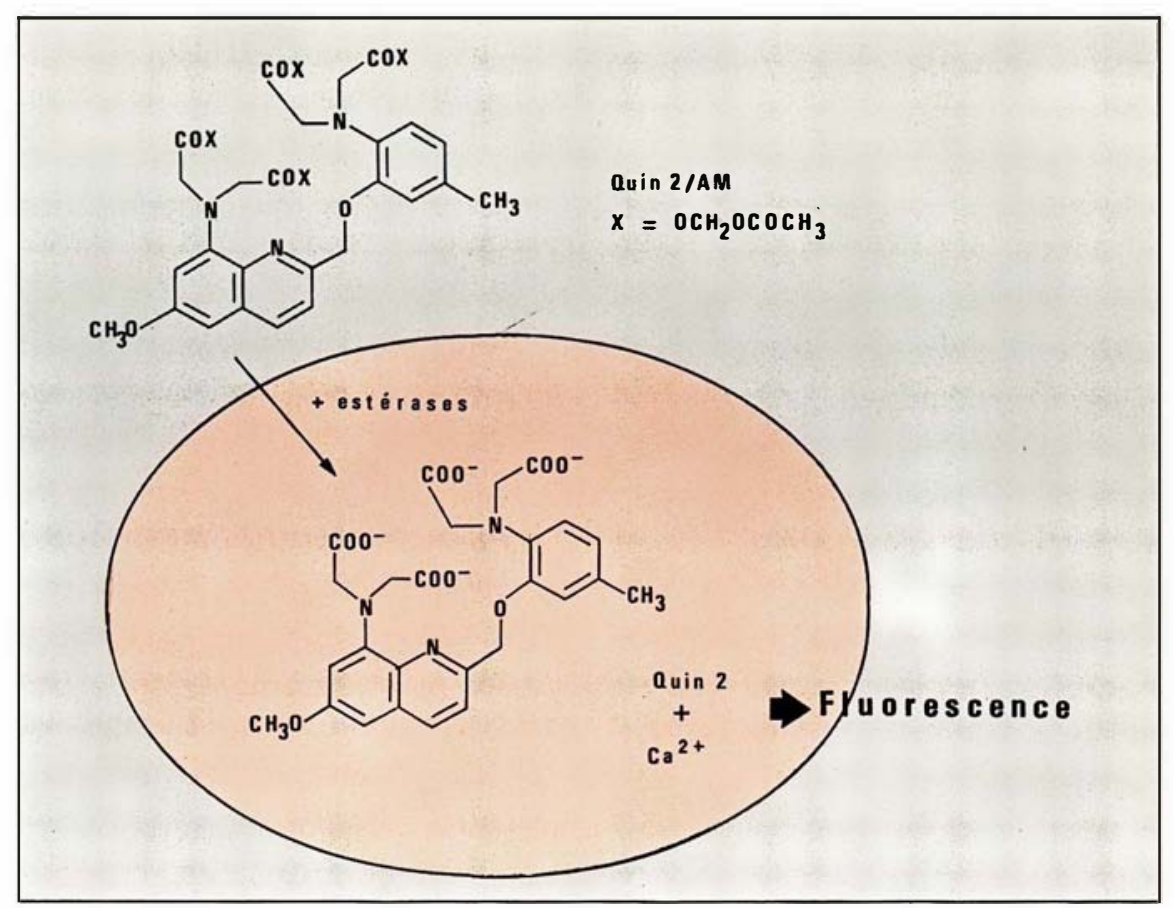

hépatocytes. Cette mesure est réalisée grâce à l'utilisation d'indicateurs photochimiques qui lient spécifiquement le $\mathrm{Ca}^{2+}$. Ces molécules sont par exemple l'aequorine qui est une protéine bioluminescente isolée à partir de la méduse Aequorea aequorea et qui émet une lumière bleue après liaison du $\mathrm{Ca}^{2+}$. D'autres molécules comme l'arsenazo III doivent être excitées et absorbent la lumière dans certaines longueurs d'onde spécifiques lorsqu'elles sont complexées avec le $\mathrm{Ca}^{2+}$. Les indicateurs fluorescents synthétisés par $\mathrm{R}$. Tsien [3] comme le quin 2 puis le fura 2 et l'indo 1, ont acquis une grande popularité au cours de ces dernières années. Ces indicateurs lient le $\mathrm{Ca}^{2+}$ avec une grande affinité comparativement à d'autres ions comme $\mathrm{Na}^{+}, \mathrm{Mg}^{2+}$ ou $\mathrm{H}^{+}$qui existent en quantité importante dans les cellules. Les complexes indicateurs- $\mathrm{Ca}$, dont la constante de dissociation est comprise entre 100 et $300 \mathrm{nM}$, deviennent fluorescents lorsqu'ils sont excités à une ou deux longueurs d'onde données. L'émission de fluorescence du complexe est une fonction saturable dépendant du $\mathrm{Ca}^{2+}$. On comprend alors que si une molécule comme le quin 2 , le fura 2 ou l'indo 1 est introduite dans des cellules, la concentration de $\mathrm{Ca}^{2+}$ libre du cytosol pourra être facilement quantifiée en mesurant la fluorescence émise. Ces indicateurs ne traversent pas la membrane plasmique et doivent être introduits dans les cellules sous forme estérifiée, diffusant à travers la membrane. Dans le cýtosol, les liaisons esters sont hydrolysées par les estérases présentes naturellement dans la cellule, et les molécules recouvrent leur affinité de liaison pour le $\mathrm{Ca}^{2+}$. Un exemple est montré pour le quin 2 dans la figure 1. La concentration de $\mathrm{Ca}^{2+}$ déterminée au moyen de marqueurs photochimiques varie de 50 à $200 \mathrm{nM}$ selon les types cellulaires. Dans le cas particulier des hépatocytes la concentration de $\mathrm{Ca}^{2+}$ dans le cytosol est de 100 à 200 nM [4]. Puisque la concentration de $\mathrm{Ca}^{2+}$ dans le sang ou le fluide interstitiel est de l'ordre de quelques millimoles/l, il existe un important gradient de concentration de $\mathrm{Ca}^{2+}$ (environ $10^{4}$ ) entre les deux faces de la membrane plasmique. C'est l'existence même de ce gradient qui 
fait $\mathrm{du} \mathrm{Ca}^{2+}$ un excellent candidat pour jouer le rôle de signal amplificateur intracellulaire. En effet, une faible augmentation de la perméabilité de la membrane plasmique pour le $\mathrm{Ca}^{2+}$ entraînera une augmentation importante de la concentration de $\mathrm{Ca}^{2+}$ ionisé dans le cytosol. Mais comment un gradient de concentration aussi important peut-il être maintenu par les cellules?

\section{Flux transmembranaires de $\mathrm{Ca}^{2+}$}

Le $\mathrm{Ca}^{2+}$ extracellulaire diffuse passivement dans le cytosol à travers la membrane plasmique en suivant son gradient de concentration (figure 2). Ce mouvement est facilité par la différence de potentiel existant entre l'intérieur et l'extérieur de la cellule. Cet influx dépend : (a) de la concentration de $\mathrm{Ca}^{2+}$ dans le milieu extracellulaire ; (b) du potentiel transmembranaire ; (c) de la perméabilité de la membrane plasmique pour le $\mathrm{Ca}^{2+}$. L'efflux de $\mathrm{Ca}^{2+}$ depuis le milieu intracellulaire vers le milieu extracellulaire qui se fait contre le gradient de concentration du $\mathrm{Ca}^{2+}$ nécessite un apport d'énergie. Celle-ci provient de l'hydrolyse de l'ATP en ADP par une ATPase (ou pompe à $\mathrm{Ca}^{2+}$ ) activée par le $\mathrm{Ca}^{2+}$ du cytosol [5]. L'activité de la pompe à $\mathrm{Ca}^{2+}$ peut être dépendante de facteurs cytosoliques comme la calmoduline [6] ou, dans les hépatocytes, d'un facteur encore non identifié [7]. Des constituants de la membrane plasmique comme le phosphatidylinositol diphosphate $\left(\mathrm{PIP}_{2}\right)$, peuvent activer la pompe à $\mathrm{Ca}^{2+}[6]$. Cette observation est importante parce qu'il a été montré que les hormones mobilisant le $\mathrm{Ca}^{2+}$ diminuent la concentration de PIP $_{2}$ dans la membrane. Ainsi, la pompe à $\mathrm{Ca}^{2+}$ pourrait jouer un rôle dans la variation de la concentration de $\mathrm{Ca}^{2+}$ cytosolique observée en présence de certaines hormones ou de certains neuromédiateurs. Un autre système participe à la sortie de $\mathrm{Ca}^{2+}$ de la cellule, il s'agit de l'échangeur $\mathrm{Na}^{+} / \mathrm{Ca}^{2+}[8]$. Ce transporteur utilise le gradient électrochimique des ions $\mathrm{Na}^{+}$ pour évacuer les ions $\mathrm{Ca}^{2+}$. Le gradient de concentration en $\mathrm{Na}^{+}$ résulte lui-même de l'activité de la $\mathrm{Na}^{+} / \mathrm{K}^{+}$ATPase qui rejette le $\mathrm{Na}^{+}$de la cellule en échange avec le $\mathrm{K}^{+}$. L'échangeur $\mathrm{Na}^{+} / \mathrm{Ca}^{2+}$ est abondant dans les cellules excitables et possède une capacité importante d'évacuation $\mathrm{du} \mathrm{Ca}^{2+}$. Cependant, son rôle éventuel dans le foie n'est pas encore clairement établi.

Ainsi, l'efflux de $\mathrm{Ca}^{2+}$ de la cellule dépend du niveau de régulation de la pompe à $\mathrm{Ca}^{2+}$ et de l'échangeur $\mathrm{Na}^{+} / \mathrm{Ca}^{2+}$ qui euxmêmes dépendent de l'état physiologique de la cellule, et de la concentration de $\mathrm{Ca}^{2+}$ dans le cytosol. Dans une cellule au repos les flux transmembranaires de $\mathrm{Ca}^{2+}$, c'est-à-dire l'influx et l'efflux, sont identiques. L'équilibre entre ces flux détermine la concentration de $\mathrm{Ca}^{2}+$ dans le cytosol.

Nous avons vu que la concentration de $\mathrm{Ca}^{2+}$ ionisé est faible par rapport au $\mathrm{Ca}^{2+}$ total contenu dans la cellule. Ceci est dû au fait qu'une partie du $\mathrm{Ca}^{2+}$ est liée à des protéines solubles ou aux membranes intracellulaires. D'autre part, certains compartiments intracellulaires clos comme le réticulum endoplasmique ou les mitochondries peuvent accumuler du $\mathrm{Ca}^{2+}$.

Le réticulum endoplasmique, appelé réticulum sarcoplasmique dans les muscles, représente environ $10 \%$ du volume cellulaire et $50 \%$ des membranes cellulaires. Le $\mathrm{Ca}^{2+}$ est accumulé dans le réticulum endoplasmique par une pompe à $\mathrm{Ca}^{2+}$ qui, comme la pompe à $\mathrm{Ca}^{2}+$ de la membrane plasmique, est une ATPase. La concentration de $\mathrm{Ca}^{2+}$ qui entraîne une activation de $50 \%$ de l'activité maximale de la pompe est d'environ $0,3 \mu \mathrm{M}[9,10]$. Cette concentration est proche de la concentration de $\mathrm{Ca}^{2+}$ cytosolique, ce qui signifie que le réticulum endoplasmique accumule du $\mathrm{Ca}^{2+}$ dans une cellule au repos. Le mécanisme permettant la sortie de $\mathrm{Ca}^{2+}$ de ce compartiment n'est pas encore élucidé, mais il
10. Becker GL, Fiskum G, Lehninger AL. Regulation of free $\mathrm{Ca}^{2+}$ by liver, mitochon-
dria and endoplasmic reticulum. I Biol Chem 1980 ; 255 : 9009-12. 


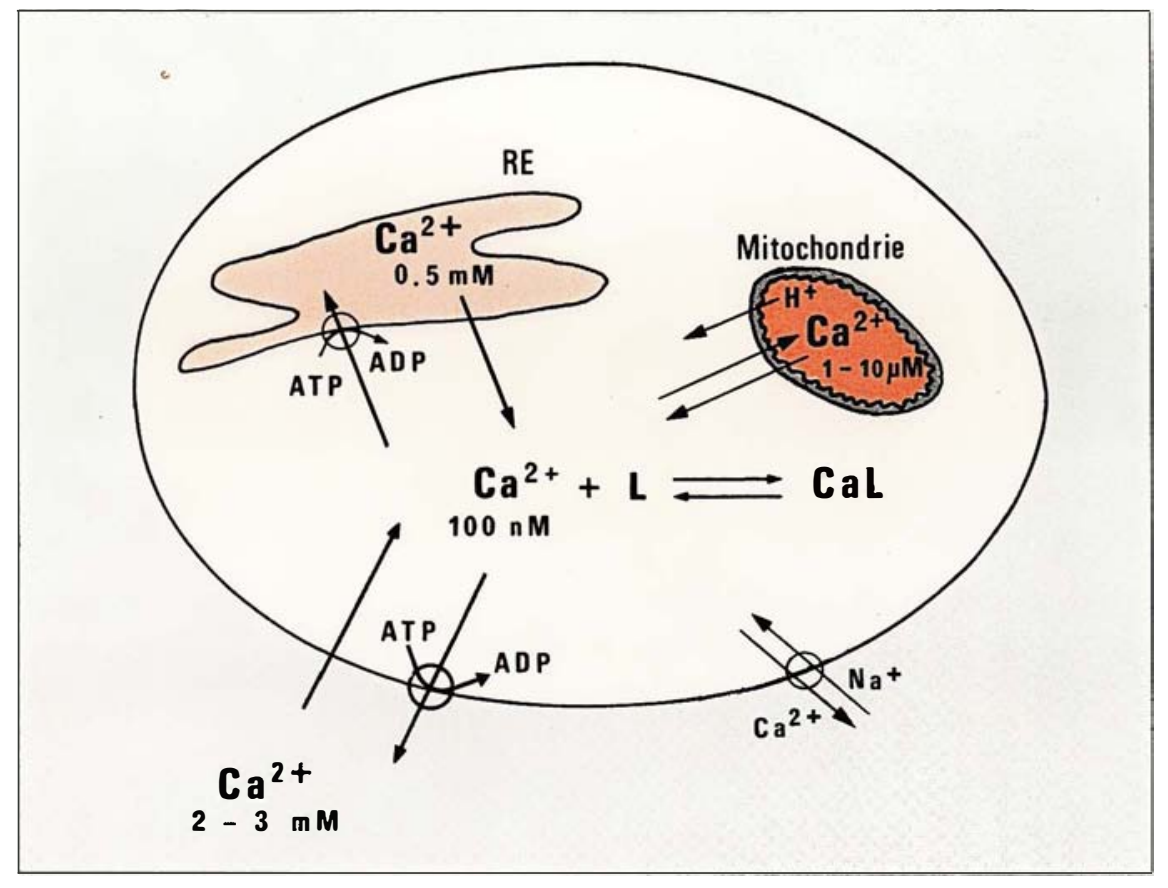

Figure 2. Homéostasie du $\mathrm{Ca}^{2}+$ cellulaire. Le $\mathrm{Ca}^{2+}$ extracellulaire traverse la membrane plasmique en suivant son gradient de concentration. Des pompes à $\mathrm{Ca}^{2}+$ rejettent le $\mathrm{Ca}^{2}+$ dans le milieu extracellulaire ou l'accumulent dans le réticulum endoplasmique (RE). Les mitochondries accumulent aussi du $\mathrm{Ca}^{2+}$ en utilisant le gradient de $\mathrm{H}^{+}$provenant du fonctionnement de la chaîne respiratoire. Le $\mathrm{Ca}^{2}+$ cytosolique peut également se fixer sur des sites anioniques intracellulaires (L). Enfin, un échangeur $\mathrm{Na}^{+} / \mathrm{Ca}^{2+}$ participe dans certaines cellules à la sortie du $\mathrm{Ca}^{2+}$ dans le milieu extracellulaire.

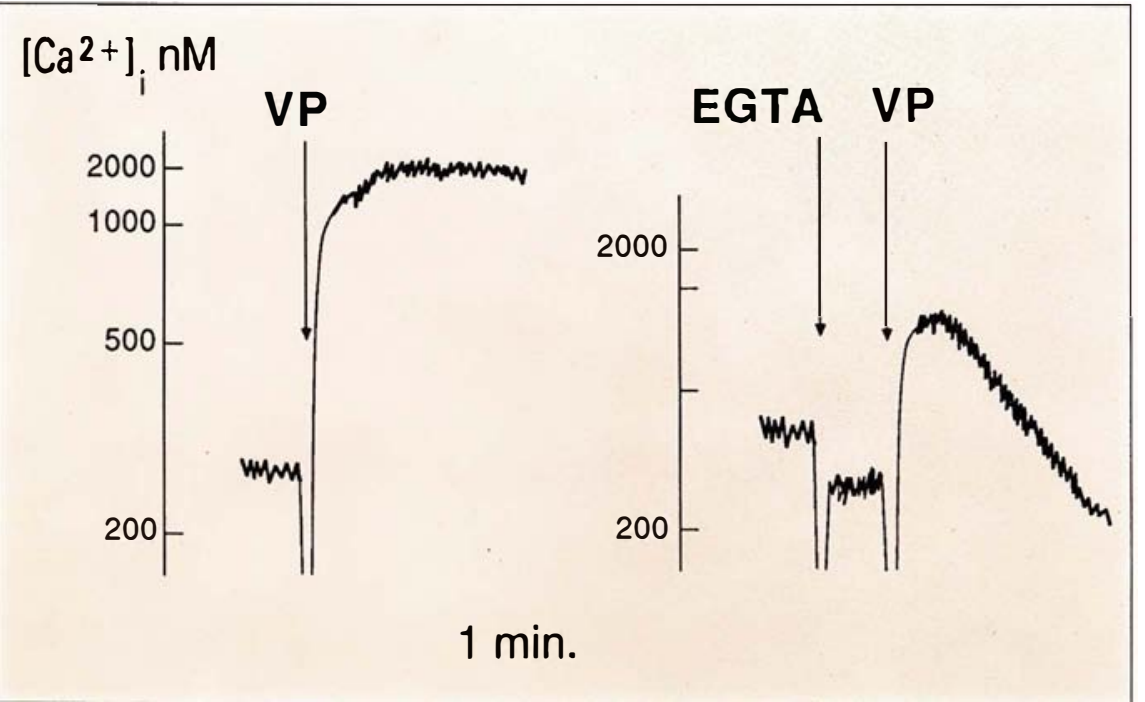

Figure 3. Enregistrement de l'effet de la vasopressine (10 nM) dans les hépatocytes de rat isolés et chargés avec le marqueur quin 2. La fluorescence de l'indicateur est calibrée en terme de $\left[\mathrm{Ca}^{2+}\right]_{i}(\mathrm{nM})$. En présence $d^{\prime} u n e$ concentration normale de $\mathrm{Ca}^{2+}$ externe $(1,8 \mathrm{mM})$, le signal indique que l'hormone augmente $\left[\mathrm{Ca}^{2+}\right]_{i}$ de 250 à $2000 \mathrm{nM}$. La réponse est soutenue. En absence de $\mathrm{Ca}^{2+}$ libre externe, condition obtenue en ajoutant du chélateur calcique EGTA $(2,4 \mathrm{mM})$, I'hormone provoque une augmentation transitoire du signal.

$\mathrm{m} / \mathrm{s} n^{\circ} 10$ vol. 3, décembre 87 est probable que le $\mathrm{Ca}^{2+}$ diffuse par des canaux spécifiques en suivant son gradient de concentration.

Les mitochondries sont des organites où s'effectue la " respiration cellulaire " permettant la transformation des substances nutritives en énergie. La chaîne respiratoire de la membrane interne des mitochondries entraîne une sortie de protons. Le gradient de protons qui en résulte fournit l'énergie nécessaire à l'accumulation de $\mathrm{Ca}^{2+}$-dans les mitochondries. Des études effectuées sur des cellules dont la membrane plasmique a été spécifiquement perméabilisée avec de la saponine, de façon à contrôler le milieu environnant les mitochondries, ont permis de constater que ces organites accumulent le $\mathrm{Ca}^{2+}$ avec une grande capacité et une faible affinité [9, 10]. Des conclusions identiques ont été obtenues à partir d'expériences effectuées sur des mitochondries isolées [10]. La concentration de $\mathrm{Ca}^{2+}$ qui entraîne une accumulation de $50 \%$ de la capacité maximale est de 10 à $20 \mu \mathrm{M}$. Cette concentration est 100 fois supérieure à la concentration de $\mathrm{Ca}^{2+}$ cytosolique d'une cellule au repos. Ceci explique le fait que les mitochondries accumulent peu de $\mathrm{Ca}^{2+}$ dans une cellule au repos. Cependant, la grande capacité d'accumulation du $\mathrm{Ca}^{2+}$ par ces organites leur permet de s'opposer aux variations importantes de la concentration intracellulaire de $\mathrm{Ca}^{2+}$.

\section{Mobilisation du $\mathrm{Ca}^{2+}$ par les hormones}

Il est possible de mesurer les variations de concentration de $\mathrm{Ca}^{2+}$ cytosolique produites par les hormones dans des cellules préalablement chargées avec du quin 2. Ainsi, dans les hépatocytes stimulés par les hormones vaso-actives comme la vasopressine ou l'angiotensine ou les agonistes $\alpha_{1}$-adrénergiques, la concentration de $\mathrm{Ca}^{2+}$ cytosolique augmente d'un niveau de base de 100 à $200 \mathrm{nM}$ à plus de $1 \mu \mathrm{M}$ en quelques secondes (figure 3) [4]. 


\section{RÉFÉRENCES}

11. Berridge MJ, Irvine RF. Inositol trisphosphate, a novel second messenger in cellular signal transduction. Nature 1984 ; 312 : 315-21.

12. Le Peuch $\mathrm{C}$. De nouveaux seconds messagers de l'action hormonale. médecine/sciences $1985 ; 1: 255-60$.

13. Streb H, Irvine RF, Berridge MJ, Schulz I. Release of $\mathrm{Ca}^{2}+$ from a non mitochondrial intracellular store in pancreatic acinar cells by inositol-1,4,5-trisphosphate. Nature 1983 ; 306 : 67-9.

14. Burgess GM, Godf rey PP, McKinney JS, Berridge $\mathrm{MJ}$, Irvine RF, Putney JW. The second messenger linking receptor activation to internal $\mathrm{Ca}^{2+}$ release in liver. Nature 1984 ; 309 : 63-6.

15. Spat A, Bradford PG, McKinney JS, Rubin RP, Putney JW. A saturable receptor for ${ }^{32} \mathrm{P}$-inositol-1,4,5-trisphosphate in hepatocytes and neutrophils. Nature $1986 ; 319$ : 514-6.

16. Dawson AP. GTP enhances inositol trisphosphate-stimulated $\mathrm{Ca}^{2}+$ release from rat liver microsomes. FEBS Lett $1985 ; 185$ : 147-50.

17. Mauger JP, Poggioli J, Guesdon F, Claret $M$. Noradrenaline, vasopressin and angiotensin increase $\mathrm{Ca}^{2}+$ influx by opening a common pool of $\mathrm{Ca}^{2}+$ channels in isolated rat liver cells. Biochem $J$ 1984; 221 : 121-7.

18. Reuter $\mathrm{H}$. Calcium channel modulation by neurotransmitters, enzymes and drugs. Nature 1983 ; 301 : 569-74.

19. Kuno M, Gardner P. Ion channels activated by inositol-1,4,5-trisphosphate in plasma membrane of human T-lymphocytes. Nature 1987 ; 326 : 301-4.

20. Irvine RF, Moor RM. Micro-injection of inositol-1,3,4,5-tetrakisphosphate activates sea urchin eggs by a mechanism dependent on external $\mathrm{Ca}^{2+}$. Biochem $J 1986 ; 240: 917-20$.

21. Von-Tscharner V, Prod'hom B, Baggiolini $M$, Reuter $H$. Ion channels in human neutrophils activated by a rise in free cytosolic calcium concentration. Nature $1986 ; 324$ :

Des expériences effectuées sur les hépatocytes isolés ont montré que la phase initiale de la réponse était due à la libération de $\mathrm{Ca}^{2+}$ d'un compartiment intracellulaire identifié comme étant le réticulum endoplasmique (figure 3). Cependant, cette réserve de $\mathrm{Ca}^{2+}$ étant de capacité limitée, elle ne permet pas de maintenir la concentration cytosolique de $\mathrm{Ca}^{2+}$ à un niveau élevé de façon soutenue. Le maintien de la réponse est dû à l'entrée de $\mathrm{Ca}^{2+}$ à partir du milieu extracellulaire.

Le fait que le signal hormonal soit reçu au niveau de la membrane plasmique par des récepteurs spécifiques et le $\mathrm{Ca}^{2+}$ libéré d'un réservoir intracellulaire, suggère l'existence d'un autre messager intracellulaire. La liaison de l'hormone sur son récepteur entraîne l'activation d'une phospholipase C qui hydrolyse le phosphatidylinositol 4,5 diphosphate ( $\mathrm{PIP}_{2}$ ) présent dans la membrane, en diacylglycérol et en inositol triphosphate $\left(\mathrm{IP}_{3}\right)[11,12]$. M. Berridge [11] a proposé que l'IP pourrait être le messager intracellulaire responsable de la mobilisation $\mathrm{du} \mathrm{Ca}^{2+}$ intracellulaire. Pour tester cette hypothèse, il faut pouvoir accéder au réticulum endoplasmique ; cette condition est obtenue en utilisant des cellules traitées avec de la saponine qui, en se fixant au cholestérol abondant dans la membrane plasmique, forme des pores qui laissent passer les ions et les molécules de taille moyenne. Ces cellules sont incubées dans un milieu dont la composition ionique ressemble à celle du milieu intracel-

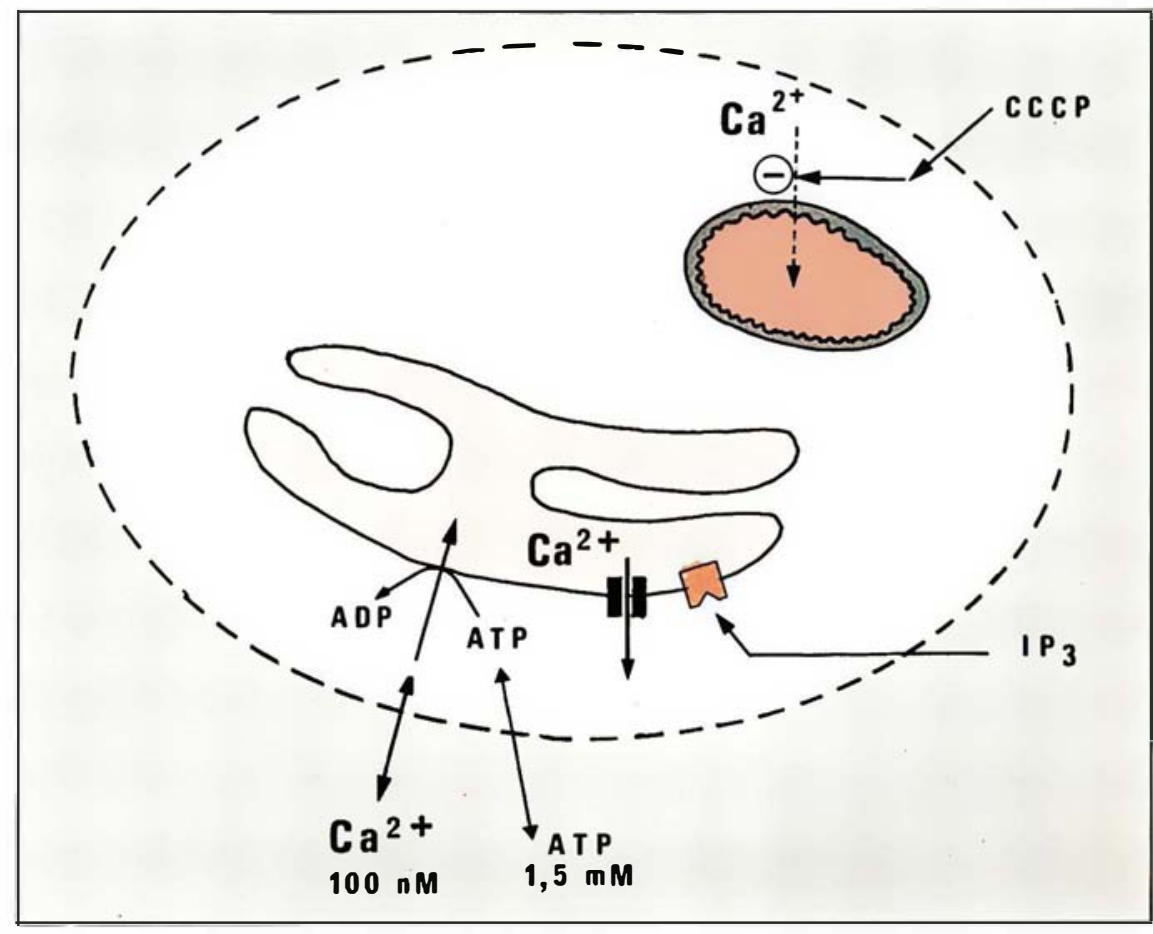

Figure 4. Perméabilisation des cellules avec la saponine. Des cellules traitées avec de la saponine sont perméables aux molécules de petite taille alors que les organites intracellulaires restent fonctionnels. Le milieu d'incubation reproduit la composition ionique du milieu intracellulaire. L'addition d'ATP dans le milieu permet l'accumulation de $\mathrm{Ca}^{2+}$ dans le réticulum endoplasmique. La perméabilisation permet à $I^{\prime} I P_{3} d^{\prime}$ accéder à son récepteur localisé sur le réticulum endoplasmique. L'entrée de $\mathrm{Ca}^{2+}$ dans les mitochondries est inhibée par des inhibiteurs mitochondriaux comme le carbonyl cyanide m-chlorophénylhydrazone (CCCP). 
lulaire et l'addition d'ATP entraîne l'accumulation de $\mathrm{Ca}^{2+}$ dans le réticulum endoplasmique (figure 4). L'addition d'IP ${ }_{3}$ provoque une perte rapide du $\mathrm{Ca}^{2+}$ contenu dans le réticulum endoplasmique (figure 5) $[13,14]$. Cet effet est indépendant de la température ce qui suggère que $\mathrm{l}^{\prime} \mathrm{IP}_{3}$ ouvre des canaux calciques et n'affecte pas les propriétés de la pompe à $\mathrm{Ca}^{2+}$. Des expériences effectuées avec du $\left.{ }^{32} \mathrm{P}\right] \mathrm{IP}_{3}$ indiquent que ce ligand se fixe sur des récepteurs spécifiques avec une constante de dissociation $\left(\mathrm{K}_{\mathrm{D}}\right)$ de $10^{-8}$ à $10^{-7} \mathrm{M}[15]$. Cependant, les mécanismes conduisant de la liaison de l'IP ${ }_{3}$ sur son récepteur à l'ouverture des canaux $\mathrm{Ca}^{2+}$ ne sont pas encore connus. Des travaux récents indiquent que le GTP pourrait être impliqué dans ce processus [16]. D'autre part, nous savons que les hormones mobilisant le $\mathrm{Ca}^{2+}$ stimulent l'influx de $\mathrm{Ca}^{2+}$ depuis le milieu extracellulaire [17]. Ainsi, dans une cellule stimulée par une hormone, deux flux de $\mathrm{Ca}^{2+}$ d'origine différente convergent vers le cytosol pour augmenter de façon considérable et soutenue la concentration de $\mathrm{Ca}^{2+}$ dans ce compartiment. Cependant, les étapes impliquées dans la stimulation de l'influx de $\mathrm{Ca}^{2+}$ par les hormones ne sont pas connues. Il semble raisonnable de supposer que la cascade d'événements dépendant de l'hydrolyse du $\mathrm{PIP}_{2}$ est impliquée, bien que la relation directe avec l'influx de $\mathrm{Ca}^{2+}$ n'ait pas encore été établie. Dans les cellules excitables, le $\mathrm{Ca}^{2+}$ entre dans la cellule par des canaux spécifiques qui s'ouvrent lorsque le potentiel transmembranaire, qui est de - 80 à - 60 millivolts, diminue ou s'inverse, c'est-à-dire lorsque la membrane se dépolarise. Une telle dépolarisation se produit sous l'action de certains neuromédiateurs. Nous savons aussi que l'AMP cyclique augmente la fréquence d'ouverture des canaux $\mathrm{Ca}^{2+}[18]$. Dans les cellules non excitables, il existe deux types différents de canaux $\mathrm{Ca}^{2+}$. Une forme présente les mêmes carac-

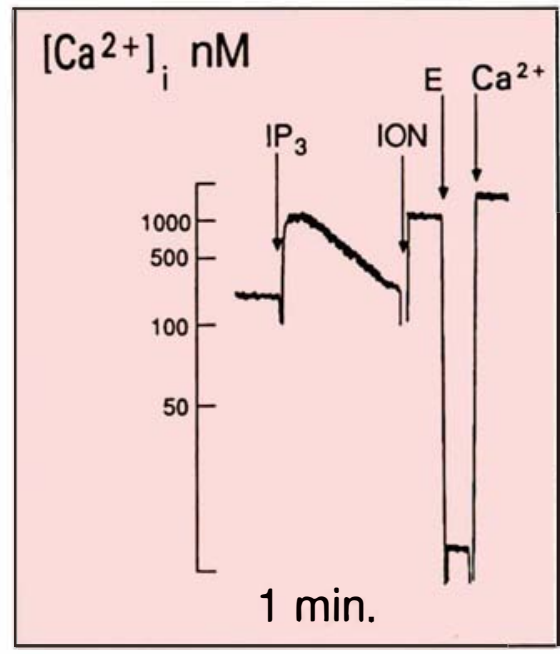

Figure 5. Enregistrement de l'effet de $I^{\prime} I P_{3}(1 \mu M)$ dans los cellulos hépatiques perméabilisées par la saponine $(50 \mu \mathrm{g} / \mathrm{ml})$ ot incubées dans un miliou dont la composition ionique reproduit celle du cytosol de la cellulo (voir texte). L'IP 3 provoque une perte rapide ( $\leq 1 s)$ du $\mathrm{Ca}^{2+}$ séquestré dans le réticulum endoplasmique. L'effet est transitoire car I'IP $P_{3}$ est rapidement dégradé par les phosphatases du cytosol, ce qui permet au réticulum endoplasmique de réaccumuler le $\mathrm{Ca}^{2+}$ perdu pendant la phase de perméabilisation. Ceci est illustré par le fait que la ionomycine (ION, $5 \mu \mathrm{M}$ ) qui est un ionophore à $\mathrm{Ca}^{2+}$ et accélère les échanges de $\mathrm{Ca}^{2+}$ à travers les membranes biologiques, libère de nouveau le $\mathrm{Ca}^{2+}$ accumulé. Expérience réalisée en présence de quin 2 (10 $\mu \mathrm{M})$ étalonné en fin d'expérience par l'addition d'EGTA $(200 \mu \mathrm{M})$ pour obtenir la fluorescence minimale et de $\mathrm{Ca}^{2}+(300 \mu \mathrm{M})$ pour obtenir la fluorescence maximale $(E=$ EGTA).

téristiques que les canaux calciques des cellules excitables, c'està-dire qu'ils s'ouvrent lorsque la membrane plasmique est dépolarisée. Ils sont en outre inhibés par les antagonistes calciques. L'autre type de canal $\mathrm{Ca}^{2+}$ trouvé dans les cellules non excitables est insensible aux inhibiteurs calciques et est activé par la liaison des hormones sur leurs récepteurs. Ces canaux appelés " receptor operated calcium channel " sont pré- sents par exemple dans les hépatocytes.

Différents mécanismes ont été proposés récemment pour expliquer l'augmentation de l'influx de $\mathrm{Ca}^{2+}$ à travers la membrane plasmique. Ainsi, par exemple, I'Ins $(1,4,5) \mathrm{P}_{3}$ active, dans les lymphocytes $T$, des canaux perméables au $\mathrm{Ca}^{2+}[19]$. L'Ins $(1,3,4,5) \mathrm{P}_{4}$, qui provient de la phosphorylation de l' $\mathrm{IP}_{3}$, pourrait aussi servir de messager intracellulaire permettant l'influx de $\mathrm{Ca}^{2+}$ à travers la membrane plasmique, comme le suggèrent des expériences effectuées sur les œufs d'oursin [20]. Enfin, dans les neutrophiles, l'augmentation de la concentration de $\mathrm{Ca}^{2+}$ cytosolique entraîne l'ouverture de canaux non spécifiques permettant l'entrée de $\mathrm{Ca}^{2+}$ à travers la membrane plasmique [21]. Ces données, pour l'instant préliminaires, conduisent à supposer que les inositols phosphates peuvent être aussi impliqués dans la mobilisation $\mathrm{du} \mathrm{Ca}^{2+}$ extracellulaire. Cependant, il faut attendre de disposer d'autres résultats expérimentaux avant de pouvoir généraliser ces mécanismes de stimulation de l'influx calcique.

Lorsque le signal hormonal cesse, l'IP $_{3}$ n'est plus produit par la phospholipase $\mathrm{C}$ et il est rapidement dégradé en inositol qui redevient disponible pour la synthèse du phosphotidylinositol. Ce processus de dégradation fait intervenir plusieurs réactions enzymatiques qui commencent par l'action d'une $\mathrm{IP}_{3}$ kinase et produit l'Ins $(1,3,4,5) \mathrm{P}_{4}$ qui, par l'action de différentes phosphomonoestérases, sera transformé en Ins $(1,3,4) \mathrm{P}_{3}$ puis en $\operatorname{IP}_{2}$, IP et inositol [22]. Il apparaît ainsi plusieurs inositols polyphosphates au sein de la cellule et leurs rôles respectifs n'ont pas encore été élucidés. La concentration d'IP dans la cellule étant revenue à son niveau de base, l'efflux de $\mathrm{Ca}^{2+}$ $\mathrm{du}$ réticulum endoplasmique revient à son niveau de repos. De même, l'influx de $\mathrm{Ca}^{2}+$ à travers la membrane plasmique diminue. Les pompes à $\mathrm{Ca}^{2+}$ de la membrane plasmique et du réticulum 
endoplasmique étant activées, elles ramènent rapidement la concentration du $\mathrm{Ca}^{2+}$ cytosolique à son niveau de repos 100 à $200 \mathrm{nM}$.

Des études menées récemment sur le foie ou les hépatocytes remettent en question plusieurs certitudes sur le mode d'action des hormones qui utilisent l'AMP cyclique comme second messager. Ainsi, par exemple, le glucagon modifie la distribution du $\mathrm{Ca}^{2+}$ dans les hépatocytes, de façon semblable à celle observée en présence d'hormones connues pour utiliser l' $\mathrm{IP}_{3}$ comme messager intracellulaire. Des études effectuées sur des hépatocytes chargés avec le quin 2 indiquent que le glucagon augmente la concentration de $\mathrm{Ca}^{2+}$ cytosolique [23]. Cette réponse est due en partie à la libération de $\mathrm{Ca}^{2+}$ depuis un réservoir intracellulaire identique à celui qui est sensible aux hormones mobilisant le $\mathrm{Ca}^{2+}$ [24]. De plus, il a été montré que le glucagon augmente l'influx de $\mathrm{Ca}^{2+}$ à travers la membrane plasmique des hépatocytes et potentialise la réponse induite par les hormones mobilisant le $\mathrm{Ca}^{2+}[25]$. Ces effets du glucagon sur la mobilisation $\mathrm{du}^{\mathrm{Ca}^{2+}}$ contenu dans le réticulum endoplasmique et la stimulation de l'influx de $\mathrm{Ca}^{2+}$ à travers la membrane plasmique, sont également observés lorsque les hépatocytes sont stimulés par un agoniste $\beta$-adrénergique comme l'isoprotérénol, ou par la forskoline qui stimule directement l'adénylcyclase. De même, l'incubation d'hépatocytes en présence d'analogues de l'AMP cyclique comme le dibutyryl AMP cyclique entraîne les mêmes effets que le glucagon sur les mouvements de $\mathrm{Ca}^{2+}$. Il apparaît ainsi que l'augmentation du contenu cellulaire en AMP cyclique provoque une libération du $\mathrm{Ca}^{2+}$ contenu dans le réticulum endoplasmique et une stimulation de l'influx de $\mathrm{Ca}^{2+}$ à travers la membrane plasmique. Un article récent [26] montre que le glucagon peut interagir avec deux types de récepteurs différents l'un étant couplé à l'adénylcyclase, l'autre entraînant l'activa- tion de la phospholipase $\mathrm{C}$ qui hydrolyse le $\mathrm{PIP}_{2}$ en $\mathrm{IP}_{3}$ et en diacylglycérol. La stimulation de ce deuxième type de récepteur peut rendre compte en partie des effets du glucagon sur la distribution et les flux de $\mathrm{Ca}^{2+}$. Cependant, il est clair que l'augmentation de la concentration intracellulaire d'AMP cyclique provoquée par l'hormone est également impliquée dans la mobilisation du $\mathrm{Ca}^{2+}$ intracellulaire et l'augmentation de l'influx de $\mathrm{Ca}^{2+}$. Le mode d'action de l'AMP cyclique reste à ce jour l'objet de controverses. Il pourrait lui aussi provoquer l'hydrolyse du $\mathrm{PIP}_{2}$ en $\mathrm{IP}_{3}$ et en DAG [27] ce qui expliquerait que l'AMP cyclique produise les mêmes effets que les hormones mobilisant le $\mathrm{Ca}^{2+}$. Mais certains laboratoires n'ont pu mettre en évidence d'accumulation d'IP $_{3}$ dans des hépatocytes stimulés par le glucagon ou incubés en présence d'analogues de l'AMP cyclique $[28,29]$.

Dans les cellules de foie, l'AMP cyclique et le $\mathrm{Ca}^{2+}$ contrôlent les mêmes fonctions métaboliques ; il est donc permis de penser que l'augmentation de la concentration de $\mathrm{Ca}^{2+}$ cytosolique provoquée par les hormones participe au développement complet de la réponse physiologique.

\section{Protéines de liaison du $\mathrm{Ca}^{2+}$}

L'augmentation de la concentration de $\mathrm{Ca}^{2+}$ dans le cytosol entraîne la modification de nombreuses fonctions cellulaires qui vont de la contraction musculaire à la sécrétion ou la régulation d'enzymes impliquées dans le métabolisme. C'est en se fixant sur des protéines spécifiques que le $\mathrm{Ca}^{2+}$ remplit son rôle de signal intracellulaire. Les protéines récepteurs des ions $\mathrm{Ca}^{2+}$ lient le $\mathrm{Ca}^{2+}$ avec une haute affinité $\left(\mathrm{K}_{\mathrm{D}}=10^{-8}\right.$ à $\left.10^{-6} \mathrm{M}\right)$ et cette liaison produit des changements de conformation. Il en résulte une modification de leurs fonctions, ou de leur capacité à interagir avec d'autres protéines pour modifier leur fonction.
31. Claret M, Binet A. Mécanisme d'action des hormones mobilisant le calcium dans les hépatocytes de mammiferes. $J$ Physiol (Paris) $1984 ; 79: 120-8$. 
Parmi les enzymes qui voient leur activité directement modifiée par le $\mathrm{Ca}^{2+}$, on peut noter la protéine kinase $\mathrm{C}$ ou des enzymes mitochondriales comme la pyruvate déshydrogénase ou ${ }^{\prime} \alpha$ cétoglutarate déshydrogénase [30]. D'autres protéines n'ont pas d'activité enzymatique intrinsèque. Cependant, lorsqu'elles lient le $\mathrm{Ca}^{2+}$, elles subissent un changement de conformation leur permettant d'interagir avec d'autres protéines et aussi de modifier certaines fonctions de la cellule. Parmi ces protéines, on notera la troponine $\mathrm{C}$ qui joue un rôle important dans la contraction musculaire, la parvalbumine qu'on trouve dans le muscle squelettique, la chaîne légère de la myosine et enfin la calmoduline. Cette dernière, présente dans tous les types cellulaires, est capable d'agir sur un grand nombre d'enzymes dans la cellule. La calmoduline peut modifier le métabolisme des nucléotides cycliques en agissant sur l'adénylate-cyclase, la guanylate-cyclase ou la phosphodiestérase qui dégrade l'AMP cyclique. Elle stimule aussi la $\mathrm{Ca}^{2+}$ ATPase de la membrane plasmique dans plusieurs types cellulaires, et active différentes protéines kinases.

La calmoduline est une simple chaîne polypeptidique composée de 48 acides aminés qui peut fixer quatre ions $\mathrm{Ca}^{2+}$. Cependant, les quatre sites de liaison du $\mathrm{Ca}^{2+}$ ne sont pas tous équivalents et il est probable que l'occupation des premiers sites modifie l'affinité des autres sites de liaison $\mathrm{du} \mathrm{Ca}^{2+}$. Cette plasticité de la sensibilité de la calmoduline pour le $\mathrm{Ca}^{2+}$ lui permet d'agir sur différentes fonctions cellulaires. Ainsi selon la concentration du $\mathrm{Ca}^{2+}$ cytosolique, la calmoduline interagit avec des protéines différentes. La calmoduline peut se trouver sous forme soluble dans le cytosol d'une cellule au repos ou bien faire partie intégrante d'un complexe enzymatique comme c'est le cas pour la phosphorylase kinase. Cette enzyme est constituée de quatre sous-unités appelées $\alpha, \beta$, $\gamma$ et $\delta$. La sous-unité $\gamma$ est l'unité catalytique et la sous-unité $\delta$ est identique à la calmoduline et rend l'enzyme sensible au $\mathrm{Ca}^{2+}$. $\mathrm{La}$ phosphorylase kinase existe sous deux formes stables, phosphorylée ou non phosphorylée. L'enzyme phosphorylée par la protéine kinase dépendante de l'AMP cyclique a une constante de demiactivation pour le $\mathrm{Ca}^{2+}$ de $0,5 \mu \mathrm{M}$ alors que l'enzyme non phosphorylée a une constante de $30 \mu \mathrm{M}$. La phosphorylase kinase activée entraîne la phosphorylation de la glycogène phosphorylase $b$ inactive pour la transformer en forme a active [31]. Parallèlement à l'activation de la phosphorylase kinase, le complexe calmoduline$\mathrm{Ca}$ provoque une inhibition de la glycogène synthase par l'intermédiaire de la glycogène synthase kinase. Cette séquence d'événements permet au muscle de disposer du glucose nécessaire pour lui fournir de l'énergie et au foie de libérer le glucose dans le flux sanguin.

L'utilisation du $\mathrm{Ca}^{2+}$ comme signal intracellulaire ne se limite pas à la régulation de fonctions telles que le métabolisme du glucose, la sécrétion ou la division cellulaire mais permet aussi le développement de processus très rapides tels que la libération de neurotransmetteurs ou la contraction musculaire. Un tel rôle du $\mathrm{Ca}^{2+}$ n'est possible que parce que la concentration du $\mathrm{Ca}^{2+}$ cytosolique est très faible par rapport aux concentrations de $\mathrm{Ca}^{2+}$ dans les différents organites intracellulaires ou le milieu extracellulaire. Ainsi de faibles variations des flux de $\mathrm{Ca}^{2+}$ à travers la membrane plasmique ou la membrane du réticulum endoplasmique entraînent des variations rapides et importantes de la concentration de $\mathrm{Ca}^{2+}$ cytosolique. Cependant, les mécanismes mis en jeu au cours d'une réponse hormonale qui permettent l'augmentation de l'influx de $\mathrm{Ca}^{2+}$ à travers la membrane plasmique ou de l'efflux de $\mathrm{Ca}^{2+}$ du réticulum endoplasmique stimulé par l' $^{\prime} \mathrm{IP}_{3}$, ne sont pas encore parfaitement élucidés

\section{Summary}

Several hormones or neurotransmitters use $\mathrm{Ca}^{2+}$ as intracellular signal to regulate metabolic pathways like glycogenolysis. The cytosolic $\mathrm{Ca}^{2+}$ concentration is maintained at low level (100-200) nM by means of a $\mathrm{Ca}^{2+}$ ATPase located in the plasma membrane, which actively pumps $\mathrm{Ca}^{2+}$ out of the cell. Two major intracellular organelles, the endoplasmic reticulum and, to a lesser extent, the mitochondria also actively accumulate $\mathrm{Ca}^{2+}$. The $\mathrm{Ca}^{2+}$ mobilizing hormones induce the release of the $\mathrm{Ca}^{2+}$ contained in the endoplasmic reticulum by formation of the recently discovered second messenger Ins(1,4,5)-trisphosphate. These hormones also stimulate the $\mathrm{Ca}^{2+}$ influx of extracellular $\mathrm{Ca}^{2+}$. The $\mathrm{Ca}^{2+}$ release from the endoplasmic reticulum and cell $\mathrm{Ca}^{2}$ influx contribute to increase the $\mathrm{Ca}^{2+}$ concentration in the cytosol. The resulting activation of cell $\mathrm{Ca}^{2+}$ binding proteins like calmodulin induce the physiological response.

\section{TIRÉS À PART}

M. Claret : unité de recherches de physiologie et pharmacologie cellulaire, Inserm U 274, bâtiment 443, université Paris-Sud, 91405 Orsay Cedex, France. 